\title{
KOROVKIN-TYPE RESULTS ON CONVERGENCE OF SEQUENCES OF POSITIVE LINEAR MAPS ON FUNCTION SPACES
}

\author{
MALIHEH HOSSEINI AND JUAN J. FONT
}

\begin{abstract}
In this paper we deal with the convergence of sequences of positive linear maps to a (not assumed to be linear) isometry on spaces of continuous functions. We obtain generalizations of known Korovkin-type results and provide several illustrative examples.
\end{abstract}

\section{INTRODUCTION}

One of the most impressive results in approximation theory is, without doubt, Korovkin's theorem on convergence of positive linear operators on a space of continuous functions. More explicitly, Korovkin's theorem (often called Korovkin's first theorem) states that if a sequence $\left\{T_{n}\right\}$ of positive linear maps on $C_{\mathbb{R}}[0,1]$ converges to the identity operator on the quadratic polynomials, then $T_{n} f$ converges to $f$ for all $\left.f \in C_{\mathbb{R}}[0,1]([]]\right)$. This result arose from a generalization of the well-known proof of Weierstrass's approximation theorem given by S. Bernstein. Its strength and simplicity have produced, as it is clearly imaginable, a wide range of applications and generalizations. One of them deals with substituting the identity operator by other operators and the closed interval $[0,1]$ by other spaces. Others center on finding subsets of function spaces, known as Korovkin sets or test functions, which guarantee that the convergence of a sequence of positive linear maps holds on the whole space provided it holds on them. For more details and other aspects of this topic, we refer to the monographs [2, 6], the recent survey paper by Altomare [1, and the references therein.

Let $X$ and $Y$ be compact Hausdorff spaces, $M$ be a unital subspace of $C(X)$, and $S$ be a function space included in $M$. In [7, the authors studied the convergence of a sequence of unital linear contractions towards a fixed linear isometry. Indeed, they proved that, under certain assumptions, if each $T_{n}: M \longrightarrow C(Y)(n \in \mathbb{N})$ is a unital linear contraction and $T_{\infty}: M \longrightarrow C(Y)$ is a linear isometry such that $\left\{T_{n} f\right\}$ converges to $T_{\infty} f$ for all $f \in S$, then $\left\{T_{n} f\right\}$ converges to $T_{\infty} f$ for all $f \in M$, not only pointwise but also uniformly. In this paper we deal with the convergence of sequences of (not necessarily contractions) positive linear maps to a (not assumed to be linear) isometry on spaces of continuous functions by combining ideas given in [7] and in the original proof

2010 Mathematics Subject Classification. Primary 41A36; Secondary 46E15.

Key words and phrases: Function space, Korovkin's theorem, Choquet boundary, positive linear map.

J.J. Font is supported by Spanish Government grant MTM2016-77143-P (AEI/FEDER, UE) and Generalitat Valenciana (Projecte GV/2018/110). 
of Korovkin's theorem. In particular, we obtain proper generalizations of [7, Theorems 3.1 and 4.1] and of several classical Korovkin-type results, and provide several illustrative examples.

\section{Preliminaries}

For any compact Hausdorff space $X$, let $C(X)$ denote the space of continuous real or complexvalued functions on $X$, equipped with the uniform norm $\|\cdot\|$. Note that we write $C_{\mathbb{R}}(X)$ instead of $C(X)$ when we want to consider only real-valued case. A unital subspace $S$ of $C(X)$ is called a function space on $X$ if $S$ separates the points of $X$ in the sense that for each $x, x^{\prime} \in X$ with $x \neq x^{\prime}$ there exists a function $f \in S$ such that $f(x) \neq f\left(x^{\prime}\right)$.

Let $S$ be a subspace of $C(X)$, which we always assume to be linear. We denote by $\mathcal{B}_{S^{*}}$ the closed unit ball of the dual space of $(S,\|\cdot\|)$. A nonempty subset $E$ of $X$ is called a boundary for $S$ if each function in $S$ attains its maximum modulus within $E$. The Choquet boundary $C h(S)$ of $S$ is the non-empty set of all points $x \in X$ for which $\delta_{x}$, the evaluation functional at $x$, is an extreme point of the closed unit ball $\mathcal{B}_{S^{*}}$. Namely, we have $\operatorname{ext}\left(\mathcal{B}_{S^{*}}\right)=\mathbb{T} C h(S)=\{\alpha x: \alpha \in \mathbb{T}$ and $x \in C h(S)\}$, where $\mathbb{T}=\{z \in \mathbb{C}:|z|=1\}$. It is known that $C h(S)$ is a boundary for $S$. In particular, one can obtain the following remark immediately:

Remark 2.1. If for each $x \in X$ there is a function $h \in S$ such that $h(x)=1$ and $|h(y)|<1$ for any $y \neq x$, then $C h(S)=X$. For example, as in Korovkin's original theorem, if we assume $X=[0,1]$ and $S=\operatorname{Span}\left\{1, x, x^{2}\right\}$, then $h(x):=1-(x-a)^{2}, a \in[0,1]$, yields $C h(S)=[0,1]$.

In the sequel, unless otherwise stated, it is assumed that $X$ and $Y$ are compact Hausdorff spaces, $M$ is a self-conjugate subspace of $C(X)$ in the sense that $\bar{f} \in M$ whenever $f \in M$, and $S$ is a function space included in $M$.

A linear map $T: M \longrightarrow C(Y)$ is called positive if $T f \geq 0$ holds for all $f \geq 0$.

Let $f, f_{1}, f_{2}, \ldots \in C(X)$ and $X_{0} \subseteq X$. If $\left\{f_{n}\right\}$ converges pointwise to $f$ on $X_{0}$, we write $f_{n} \longrightarrow f$ on $X_{0}$. Also, we omit $X_{0}$ when $X_{0}=X$.

Given $f, g \in C(X)$, we shall write $f \otimes 1+1 \otimes g$ to denote the function in $C(X \times X)$ such that $(f \otimes 1+1 \otimes g)\left(x, x^{\prime}\right):=f(x)+g\left(x^{\prime}\right)$. Furthermore, if $T, T^{\prime}: S \subseteq C(X) \longrightarrow C(Y)$, then we set $\left(T \otimes T 1 T^{\prime}\right)(f \otimes 1+1 \otimes g)(y):=T f(y)+T 1(y) T^{\prime} g(y)$ for all $f, g \in S$ and $y \in Y$.

Finally let us state the following lemma which is used in the proofs of our results.

Lemma 2.2. [5, Theorem 2.2.6] Let $S$ be a function space on $X$ and $x_{0} \in X$. Then $x_{0} \in C h(S)$ if and only if for any $\alpha, \beta \in(0, \infty)$ with $\alpha<\beta$ and any open neighborhood $U$ of $x_{0}$, there is a function $f \in S$ such that $\operatorname{Re} f \leq 0$ on $X, \operatorname{Re} f<-\beta$ on $U^{c}$ and $\operatorname{Re} f\left(x_{0}\right)>-\alpha$. 


\section{Results}

Theorem 3.1. Suppose that $\left\{T_{n}\right\}$ is a sequence of positive linear maps from $M$ into $C(Y)$, and $T_{\infty}$ is an isometry from $M$ onto a subspace $T_{\infty}(M)$ of $C(Y)$.

(a) If $T_{n} f \longrightarrow T_{\infty} f$ for all $f \in S$, then $T_{n} f \longrightarrow T_{\infty} f$ on $C h\left(T_{\infty}(S)\right)$ for all $f \in M$.

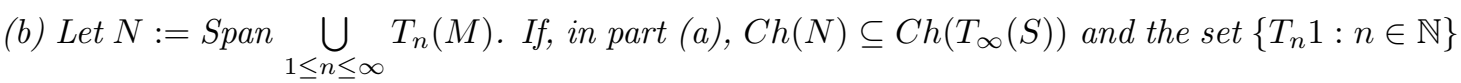
is bounded, then $T_{n} f \longrightarrow T_{\infty} f$ for all $f \in M$.

Proof. We will base the proof of (a) through the following steps.

Step 1. For each triple of distinct points $x, x^{\prime}, z \in C h(M)$, there exists a function $h \in M$ such that $|h(x)| \neq\left|h\left(x^{\prime}\right)\right|$ and $h(z)=0$.

Since $M$ is a self-conjugate function space we can find a real-valued function $f \in M$ such that $f(x)=1$ and $f\left(x^{\prime}\right)=0$. Now we consider the following cases based on the value of $f$ at $z$ :

- $f(z)=1$. Clearly, $h=1-f$ is the desired function.

- $f(z) \neq 1, \frac{1}{2}$. Take $h=f-f(z)$.

- $f(z)=\frac{1}{2}$. In this case we choose a non-negative function $g$ in $M$ with $g(x), g\left(x^{\prime}\right)>3$ and $g(z)<\frac{1}{2}$, by Lemma 2.2. If $g\left(x^{\prime}\right)-g(x)=2$, then $h=g-g(z)$ is the desired function. Otherwise, we can see that $h=2 f+g-g(z)-1$ satisfy the requested properties.

Step 2. $T_{\infty}$ is a linear isometry.

Note that $T_{\infty} 0=\lim T_{n} 0=0$. Then according to the Mazur-Ulam theorem [10, $T_{\infty}$ is a reallinear isometry. Hence now we only need to consider the complex case. Let us point out that $T_{\infty} 1=\lim T_{n} 1 \geq 0$. Taking into account Step 1, from [9, Theorem 2.3] it follows that $T_{\infty} 1=1$ and there exist a (possibly empty) clopen subset $K$ of $C h\left(T_{\infty}(M)\right)$, and a continuous surjective map $\phi: C h\left(T_{\infty}(M)\right) \longrightarrow C h(M)$ such that for all $f \in M$,

$$
T_{\infty} f= \begin{cases}f \circ \phi & \text { on } K, \\ \overline{f \circ \phi} & \text { on } C h\left(T_{\infty}(M)\right) \backslash K .\end{cases}
$$

But $T_{\infty} i=\lim T_{n} i=i \lim T_{n} 1=i T_{\infty} 1=i$, which implies that $K=C h\left(T_{\infty}(M)\right)$. Hence taking into account that $C h\left(T_{\infty}(M)\right)$ is a boundary for $T_{\infty}(M)$, we deduce that $T_{\infty}$ is a linear isometry.

Step 3. For each $f \in M, T_{n} f \longrightarrow T_{\infty} f$ on $C h\left(T_{\infty}(S)\right)$.

By [7, Lemma 2.5] (or [3, Corollary 3.2]), there is a continuous surjection $\varphi: C h\left(T_{\infty}(S)\right) \longrightarrow$ $C h(S)$ such that

$$
T_{\infty} f(y)=f(\varphi(y)) \quad\left(f \in S, y \in C h\left(T_{\infty}(S)\right)\right) .
$$


Let $f \in M$ and $\epsilon>0$. Then we can define a function in $C(X \times X)$ as $F:=f \otimes 1-1 \otimes f$. Clearly, $F=0$ on the subset $\triangle_{X}=\{(x, x): x \in X\}$ of $X \times X$. Then there is an open neighborhood $U$ of $\triangle_{X}$ with $|F|<\epsilon$ on $U$.

Let $y^{\prime} \in C h\left(T_{\infty}(S)\right)$ and $x^{\prime}=\varphi\left(y^{\prime}\right)$. Choose an open neighborhood $V_{x^{\prime}}$ of $x^{\prime}$ such that $V_{x^{\prime}} \times V_{x^{\prime}} \subseteq$ $U$. By Lemma 2.2 we find a function $f_{y^{\prime}} \in S$ such that

$$
\operatorname{Re} f_{y^{\prime}} \geq 0 \text { on } X, \operatorname{Re} f_{y^{\prime}} \geq 1 \text { on } V_{x^{\prime}}^{c}, \operatorname{Re} f_{y^{\prime}}\left(x^{\prime}\right)<\epsilon \text {. }
$$

Put $F_{y^{\prime}}=f_{y^{\prime}} \otimes 1+1 \otimes f_{y^{\prime}}$. It is clear that $\operatorname{Re} F_{y^{\prime}} \geq 0$ on $X \times X$ and $\operatorname{Re} F_{y^{\prime}} \geq 1$ on $U^{c}$. Hence we have

$$
\operatorname{Re} F \leq\|F\| \leq\|F\| \operatorname{Re} F_{y^{\prime}} \text { on } U^{c},
$$

which yields $|\operatorname{Re} F| \leq 1 \otimes \epsilon+\|F\| \operatorname{Re} F_{y^{\prime}}$ on $X \times X$. In other words,

$$
-\left(1 \otimes \epsilon+\|F\| \operatorname{Re} F_{y^{\prime}}\right) \leq \operatorname{Re} F \leq 1 \otimes \epsilon+\|F\| \operatorname{Re} F_{y^{\prime}} \text { on } X \times X .
$$

Hence for each $y \in X$ we get

$$
-\epsilon-2\|F\| \operatorname{Re} f_{y^{\prime}}-\|F\| \operatorname{Re} f_{y^{\prime}}(y)+\operatorname{Re} f(y) \leq \operatorname{Re} f-\|F\| \operatorname{Re} f_{y^{\prime}} \leq \epsilon+\|F\| \operatorname{Re} f_{y^{\prime}}(y)+\operatorname{Re} f(y) .
$$

Since $\left\{T_{n}\right\}$ is a sequence of linear positive maps, it follows that

$$
\begin{gathered}
-2\|F\| T_{n}\left(\operatorname{Re} f_{y^{\prime}}\right)+\left(-\epsilon-\|F\| \operatorname{Re} f_{y^{\prime}}(y)+\operatorname{Re} f(y)\right) T_{n} 1 \leq T_{n}(\operatorname{Re} f)-\|F\| T_{n}\left(\operatorname{Re} f_{y^{\prime}}\right) \leq \\
T_{n} 1\left(\epsilon+\|F\| \operatorname{Re} f_{y^{\prime}}(y)+\operatorname{Re} f(y)\right)
\end{gathered}
$$

for each $y \in X$. Now, from the representation of $T_{\infty}$ on $M$ (Step 2), we deduce that

$$
\begin{gathered}
-2\|F\| T_{n}\left(\operatorname{Re} f_{y^{\prime}}\right)(z)+T_{\infty}\left(-\epsilon-\|F\| \operatorname{Re} f_{y^{\prime}}+\operatorname{Re} f\right)\left(z^{\prime}\right) T_{n} 1(z) \leq T_{n}(\operatorname{Re} f)(z)-\|F\| T_{n}\left(\operatorname{Re} f_{y^{\prime}}\right)(z) \leq \\
T_{n} 1(z) T_{\infty}\left(\epsilon+\|F\| \operatorname{Re} f_{y^{\prime}}+\operatorname{Re} f\right)\left(z^{\prime}\right)
\end{gathered}
$$

for any $z \in Y$ and $z^{\prime} \in C h\left(T_{\infty}(M)\right)$. Thus, again since $T_{\infty} 1=1, T_{\infty}$ is a positive linear map and also $C h\left(T_{\infty}(M)\right)$ is a boundary for $T_{\infty}(M)$, it is observed that the above relation holds for all $z, z^{\prime} \in Y$. Therefore, especially we get

$-\|F\| T_{n}\left(\operatorname{Re} f_{y^{\prime}}\right)-T_{n} 1 T_{\infty}\left(\epsilon+\|F\| \operatorname{Re} f_{y^{\prime}}\right) \leq T_{n}(\operatorname{Re} f)-T_{n} 1 T_{\infty}(\operatorname{Re} f) \leq T_{n} 1 T_{\infty}\left(\epsilon+\|F\| \operatorname{Re} f_{y^{\prime}}\right)+\|F\| T_{n}\left(\operatorname{Re} f_{y^{\prime}}\right)$

on $Y$. Rewriting the above inequality adopted to our notation in Section 2 we have

$$
-\left(T_{n} \otimes T_{n} 1 T_{\infty}\right)\left(1 \otimes \epsilon+\|F\| \operatorname{Re} F_{y^{\prime}}\right) \leq\left(T_{n} \otimes T_{n} 1 T_{\infty}\right)(\operatorname{Re} F) \leq\left(T_{n} \otimes T_{n} 1 T_{\infty}\right)\left(1 \otimes \epsilon+\|F\| \operatorname{Re} F_{y^{\prime}}\right),
$$

equivalently,

$$
\left|\left(T_{n} \otimes T_{n} 1 T_{\infty}\right)(\operatorname{Re} F)\right| \leq\left(T_{n} \otimes T_{n} 1 T_{\infty}\right)\left(1 \otimes \epsilon+\|F\| \operatorname{Re} F_{y^{\prime}}\right) .
$$


Consequently, from the fact that each $T_{n}$ is a positive linear map and the representation of $T_{\infty}$, it follows that

$$
\begin{aligned}
\left|\operatorname{Re}\left(T_{n} \otimes T_{n} 1 T_{\infty}\right)(F)\right| & =\left|\operatorname{Re} T_{n} f-\operatorname{Re}\left(T_{n} 1 T_{\infty} f\right)\right| \\
& =\left|T_{n}(\operatorname{Re} f)-T_{n} 1 T_{\infty}(\operatorname{Re} f)\right| \\
& =\left|\left(T_{n} \otimes T_{n} 1 T_{\infty}\right)(\operatorname{Re} F)\right| \\
& \leq\left(T_{n} \otimes T_{n} 1 T_{\infty}\right)\left(1 \otimes \epsilon+\|F\| \operatorname{Re} F_{y^{\prime}}\right) \\
& =\left(T_{n} \otimes T_{n} 1 T_{\infty}\right)(1 \otimes \epsilon)+\left(T_{n} \otimes T_{n} 1 T_{\infty}\right)\left(\|F\| \operatorname{Re} F_{y^{\prime}}\right) \\
& =\epsilon T_{n} 1+\|F\|\left(T_{n}\left(\operatorname{Re} f_{y^{\prime}}\right)+T_{n} 1 T_{\infty}\left(\operatorname{Re} f_{y^{\prime}}\right)\right) \\
& =\epsilon T_{n} 1+\|F\|\left(\operatorname{Re} T_{n} f_{y^{\prime}}+T_{n} 1 \operatorname{Re} T_{\infty} f_{y^{\prime}}\right) \\
& \leq \epsilon T_{n} 1+\|F\|\left(\left|T_{n} f_{y^{\prime}}-T_{\infty} f_{y^{\prime}}\right|+T_{n} 1 \operatorname{Re} T_{\infty} f_{y^{\prime}}+\operatorname{Re} T_{\infty} f_{y^{\prime}}\right),
\end{aligned}
$$

which is to say,

$$
\left|\operatorname{Re}\left(T_{n} \otimes T_{n} 1 T_{\infty}\right)(F)\right| \leq \epsilon T_{n} 1+\|F\|\left(\left|T_{n} f_{y^{\prime}}-T_{\infty} f_{y^{\prime}}\right|+T_{n} 1 \operatorname{Re} T_{\infty} f_{y^{\prime}}+\operatorname{Re} T_{\infty} f_{y^{\prime}}\right)
$$

Thus, from the latter inequality, the representation of $T_{\infty}$ and for any sufficiently large integer $n$, we get

$$
\begin{aligned}
\left|\operatorname{Re} T_{n} f\left(y^{\prime}\right)-\operatorname{Re} T_{\infty} f\left(y^{\prime}\right)\right| & \leq\left|\operatorname{Re} T_{n} f\left(y^{\prime}\right)-T_{n} 1\left(y^{\prime}\right) \operatorname{Re} T_{\infty} f\left(y^{\prime}\right)\right|+\left|T_{n} 1\left(y^{\prime}\right) \operatorname{Re} T_{\infty} f\left(y^{\prime}\right)-\operatorname{Re} T_{\infty} f\left(y^{\prime}\right)\right| \\
& \leq \epsilon T_{n} 1\left(y^{\prime}\right)+\|F\|\left(\left|T_{n} f_{y^{\prime}}\left(y^{\prime}\right)-T_{\infty} f_{y^{\prime}}\left(y^{\prime}\right)\right|+T_{n} 1\left(y^{\prime}\right) \operatorname{Re} f_{y^{\prime}}\left(x^{\prime}\right)+\right. \\
& \left.\operatorname{Re} f_{y^{\prime}}\left(x^{\prime}\right)\right)+\left|\operatorname{Re} T_{\infty} f\left(y^{\prime}\right)\right|\left|T_{n} 1\left(y^{\prime}\right)-1\right| \\
& \leq 2 \epsilon+\|F\|(\epsilon+2 \epsilon+\epsilon)+\|f\| \epsilon \\
& =(2+4\|F\|+\|f\|) \epsilon
\end{aligned}
$$

Hence $\operatorname{Re} T_{n} f \longrightarrow \operatorname{Re} T_{\infty} f$ on $C h\left(T_{\infty}(S)\right)$. By replacing $f$ by $-i f$, we see that $\operatorname{Im} T_{n} f \longrightarrow \operatorname{Im} T_{\infty} f$ on $C h\left(T_{\infty}(S)\right)$. Therefore, $T_{n} f \longrightarrow T_{\infty} f$ on $C h\left(T_{\infty}(S)\right)$, which completes the proof of part (a).

(b) We first claim that $\left\|T_{n}\right\| \leq \sqrt{2}\left\|T_{n} 1\right\|$, where $\left\|T_{n}\right\|$ is the operator norm of $T_{n}$ (for each $n \in \mathbb{N}$ ). To see this, assume that $g \in M$ is real-valued and has supremum norm at most 1 . Then $-1 \leq g \leq 1$ and thus, $-T_{n} 1 \leq T_{n} g \leq T_{n} 1$, which implies that $\left\|T_{n} g\right\| \leq\left\|T_{n} 1\right\|$. In the real case, this shows that $T_{n}$ is continuous and the claim holds. In the complex case, from this argument and the fact that $M$ is self-conjugate, it easily follows that $\left\|T_{n}\right\| \leq \sqrt{2}\left\|T_{n} 1\right\|$.

Let $f \in M$. Taking into account the above claim and the boundedness of $\left\{T_{n} 1: n \in \mathbb{N}\right\}$, we deduce that the set $\left\{T_{n} f: n \in \mathbb{N}\right\}$ is bounded. Now one can follow the last part of the proof of [7, 
Theorem 3.3] to conclude that $T_{n} f \longrightarrow T_{\infty} f$ on $Y$ and we include it for completeness. Assume that $\sim$ is the equivalence relation on $Y$ defined by

$$
y \sim y^{\prime} \Leftrightarrow g(y)=g\left(y^{\prime}\right) \quad \forall g \in N .
$$

The quotient space of $Y$ by $\sim$ is denoted by $Y / \sim$, and $\hat{y}$ will stand for the image of $y \in Y$ under the canonical map $\hat{~}$ from $\mathrm{Y}$ onto $Y / \sim$. Moreover, we define $\hat{g}(\hat{y})=g(y)$ for all $g$ in $N$ and $y$ in $\hat{Y}=\{\hat{y}: y \in Y\}$. It is apparent that $\hat{N}=\{\hat{g}: g \in N\}$ is a function space on the compact space $\hat{Y}$.

By [4, Section V] and [12, Section 4], for any $y \in Y$, there exits a positive measure $\mu$ on the $\sigma$-ring of subsets of $\mathcal{B}_{\hat{N}^{*}}$ generated by $\operatorname{ext}\left(\mathcal{B}_{\hat{N}^{*}}\right)$ and the Baire subsets of $\mathcal{B}_{\hat{N}^{*}}$ which represents $\hat{y}$ and $\mu\left(\mathcal{B}_{\hat{N}^{*}}\right)=1$. From part (a), it is clear that $\widehat{T_{n} f} \longrightarrow \widehat{T_{\infty} f}$ on $C h \widehat{\left(T_{\infty}(S)\right)}$. Hence, since $\left.\operatorname{ext}\left(\mathcal{B}_{\hat{N}^{*}}\right)=\mathbb{T} C h(\hat{N}) \subseteq \mathbb{T} C h \widehat{\left(T_{\infty}(S)\right.}\right)$ and the set $\left\{T_{n} f: n \in \mathbb{N}\right\}$ is bounded, from the Lebesgue's dominated convergence theorem we get

$$
T_{n} f(y)=\widehat{T_{n} f}(\hat{y})=\int_{\mathcal{B}_{\hat{N}^{*}}} \widehat{T_{n} f} \longrightarrow \int_{\mathcal{B}_{\hat{N}^{*}}} \widehat{T_{\infty} f} d \mu=\widehat{T_{\infty} f}(\hat{y})=T_{\infty} f(y) .
$$

Therefore, $T_{n} f \longrightarrow T_{\infty} f$, as desired.

Let us recall here the famous Arzela-Ascoli theorem, which will be used in the proof of the next result.

Theorem (Arzela-Ascoli). Given a subset $A$ of $C(X)$, the following statements are equivalent:

(1) $A$ is a compact subset of $(C(X),\|\cdot\|)$.

(2) $A$ is closed, bounded, and equicontinuous in the sense that for each $x \in X$ and $\epsilon>0$, there exists a neighborhood $V$ of $x$ such that $|f(y)-f(x)|<\epsilon$ for all $f \in A$ and $y \in V$.

Theorem 3.2. Let $\left\{T_{n}\right\}$ be a sequence of positive linear maps from $M$ into $C(Y)$, and $T_{\infty}$ be an isometry from $M$ onto a subspace $T_{\infty}(M)$ of $C(Y)$.

(a) If $\left\{T_{n} f\right\}$ converges uniformly to $T_{\infty} f$ for all $f \in S$, then $\left\{T_{n} f\right\}$ converges uniformly to $T_{\infty} f$ on each compact subset of $C h\left(T_{\infty}(S)\right)$ for all $f \in M$.

(b) If, furthermore, either $C h\left(T_{\infty}(S)\right)$ or $C h(N)$ is compact and $C h(N) \subseteq C h\left(T_{\infty}(S)\right)$, then $\left\{T_{n} f\right\}$ converges uniformly to $T_{\infty} f$ for any $f \in M$, where $N$ is as in Theorem 3.1.

Proof. (a) As in the proof of Theorem 3.1 there is a continuous surjection $\varphi: C h\left(T_{\infty}(S)\right) \longrightarrow C h(S)$ such that for all $f \in S$,

$$
T_{\infty} f(y)=f(\varphi(y))\left(f \in S, y \in C h\left(T_{\infty}(S)\right)\right) .
$$

Suppose that $K$ is a compact subset of $C h\left(T_{\infty}(S)\right)$. Let $f \in M, y^{\prime} \in K$ and $\epsilon>0$. Put $F=$ $f \otimes 1-1 \otimes f$ and $x^{\prime}=\varphi\left(y^{\prime}\right)$. As before, we choose an open neighborhood $V_{x^{\prime}}$ of $x^{\prime}$ and a function 
$f_{y^{\prime}} \in S$ such that $\operatorname{Re} f_{y^{\prime}} \geq 0$ on $X, \operatorname{Re} f_{y^{\prime}} \geq 1$ on $V_{x^{\prime}}^{c}$ and $\operatorname{Re} f_{y^{\prime}}\left(x^{\prime}\right)<\epsilon$, and we also have

$$
\left|\operatorname{Re} T_{n} f-\operatorname{Re} T_{\infty} f\right| \leq \epsilon T_{n} 1+\|F\|\left(\left|T_{n} f_{y^{\prime}}-T_{\infty} f_{y^{\prime}}\right|+\operatorname{Re} T_{\infty} f_{y^{\prime}}+T_{n} 1 \operatorname{Re} T_{\infty} f_{y^{\prime}}\right)+\left|\operatorname{Re} T_{\infty} f\right|\left|T_{n} 1-1\right|
$$

on $Y$. Now, we prove the following claim.

Claim: The set $\left\{T_{n} f: n \in \mathbb{N}\right\}$ is equicontinuous at $y^{\prime}$.

Since $\left\{T_{n} f_{y^{\prime}}\right\}$ and $\left\{T_{n} 1\right\}$ converge uniformly to $T_{\infty} f_{y^{\prime}}$ and 1 , respectively, there is an integer $n_{0}$ such that for each $n \geq n_{0},\left\|T_{n} f_{y^{\prime}}-T_{\infty} f_{y^{\prime}}\right\|<\epsilon$ and $\left\|T_{n} 1-1\right\|<\epsilon$. On the other hand, $\operatorname{Re} T_{\infty} f_{y^{\prime}}\left(y^{\prime}\right)<\epsilon$ and so, from the continuity of $\operatorname{Re} T_{\infty} f_{y^{\prime}}$ and $T_{\infty} f$, we can choose a neighborhood $W_{y^{\prime}}$ of $y^{\prime}$ so that the inequalities $\operatorname{Re} T_{\infty} f_{y^{\prime}}<\epsilon$ and $\left|T_{\infty} f-T_{\infty} f\left(y^{\prime}\right)\right|<\epsilon$ hold on $W_{y^{\prime}}$. Hence, letting $\eta=\sup _{i \in \mathbb{N}}\left\|T_{i} 1\right\|$, for each $y \in W_{y^{\prime}}$ and $n \geq n_{0}$ we get

$$
\begin{aligned}
\left|\operatorname{Re} T_{n} f(y)-\operatorname{Re} T_{n} f\left(y^{\prime}\right)\right| & \leq\left|\operatorname{Re} T_{n} f(y)-\operatorname{Re} T_{\infty} f(y)\right|+\left|\operatorname{Re} T_{n} f\left(y^{\prime}\right)-\operatorname{Re} T_{\infty} f\left(y^{\prime}\right)\right|+ \\
& \left|\operatorname{Re} T_{\infty} f(y)-\operatorname{Re} T_{\infty} f\left(y^{\prime}\right)\right| \leq \eta \epsilon+\|F\|\left(\left|T_{n} f_{y^{\prime}}(y)-T_{\infty} f_{y^{\prime}}(y)\right|+\operatorname{Re} T_{\infty} f_{y^{\prime}}(y)+\right. \\
& \left.\eta T_{\infty} f_{y^{\prime}}(y)\right)+\|f\|\left|T_{n} 1(y)-1\right|+\eta \epsilon+\|F\|\left(\left|T_{n} f_{y^{\prime}}\left(y^{\prime}\right)-T_{\infty} f_{y^{\prime}}\left(y^{\prime}\right)\right|+\right. \\
& \left.\operatorname{Re} T_{\infty} f_{y^{\prime}}\left(y^{\prime}\right)+\eta T_{\infty} f_{y^{\prime}}\left(y^{\prime}\right)\right)+\|f\|\left|T_{n} 1\left(y^{\prime}\right)-1\right|+\left|\operatorname{Re} T_{\infty} f(y)-\operatorname{Re} T_{\infty} f\left(y^{\prime}\right)\right| \\
& \leq \eta \epsilon+\|F\|(\epsilon+\epsilon+\eta \epsilon)+\|f\| \epsilon+\eta \epsilon+\|F\|(\epsilon+\epsilon+\eta \epsilon)+\|f\| \epsilon+\epsilon \\
& =\epsilon(2 \eta+2\|f\|+4\|F\|+2 \eta\|F\|)+\epsilon .
\end{aligned}
$$

Now, from the continuity of $T_{1} f, \ldots, T_{n_{0}} f$, it follows that the set $\left\{\operatorname{Re} T_{n} f: n \in \mathbb{N}\right\}$ is equicontinuous at $y^{\prime}$. Similarly, the set $\left\{\operatorname{Im} T_{n} f: n \in \mathbb{N}\right\}$ is equicontinuous at $y^{\prime}$, and, as a consequence, $\left\{T_{n} f: n \in \mathbb{N}\right\}$ is equicontinuous at $y^{\prime}$, as claimed.

Moreover, as observed in the proof of Theorem 3.1(b), $\left\{T_{n} f: n \in \mathbb{N}\right\}$ is bounded. Therefore, from the Arzela-Ascoli theorem and Theorem 3.1(a), it follows that each subsequence $\left\{T_{n} f\right\}$ has a uniformly convergent sequence to $T_{\infty} f$ on $K$. This argument shows that $\left\{T_{n} f\right\}$ converges uniformly to $T_{\infty} f$ on the compact set $K$.

(b) When either $C h\left(T_{\infty}(S)\right)$ or $C h(N)$ is compact, then, from the above discussion, we deduce that $\left\{T_{n} f\right\}$ converges uniformly to $T_{\infty} f$ on $C h(N)$. Next, since $C h(N)$ is a boundary for $N$, it is immediately seen that $\left\{T_{n} f\right\}$ converges uniformly to $T_{\infty} f$ (on $Y$ ).

Remark 3.3. We would like to remark that the sequential version of Korovkin's theorem does not yield its net version (see [14]). However, it can be easily checked that our techniques hold true when we replace the sequence $\left\{T_{n}\right\}$ by a net of positive linear maps.

In the following corollary, we obtain the main results of [7, namely, [7, Theorem 3.3] and [7, Theorem 4.1] as consequences of Theorems 3.1 and 3.2 
Corollary 3.4. Let $M$ be a subspace of $C(X), S \subseteq M$ be a function space, $\left\{T_{n}\right\}$ be a sequence of unital linear contractions from $M$ into $C(Y), T_{\infty}$ be a linear isometry from $M$ into $C(Y)$, and $C h(N) \subseteq C h\left(T_{\infty}(S)\right)$, where $N:=$ Span $\bigcup_{1 \leq n \leq \infty} T_{n}(M)$.

(a) If $T_{n} f \longrightarrow T_{\infty} f$ for all $f \in S$, then $T_{n} f \longrightarrow T_{\infty} f$ for all $f \in M$.

(b) If $\left\{T_{n} f\right\}$ converges uniformly to $T_{\infty} f$ for all $f \in S$, then $\left\{T_{n} f\right\}$ converges uniformly to $T_{\infty} f$ on each compact subset of $C h\left(T_{\infty}(S)\right)$ for any $f \in M$. If, furthermore, $C h\left(T_{\infty}(S)\right)$ or $C h(N)$ is compact, then $\left\{T_{n} f\right\}$ converges uniformly to $T_{\infty} f$ for all $f \in M$.

Proof. In the context of real-valued function spaces, since every linear map $\mathcal{T}$ with $\|\mathcal{T}\|=\mathcal{T}(1)=1$ is positive ([13), the result follows immediately from Theorems 3.1 and 3.2. Now let us consider the complex case. We note that

$$
M+\bar{M}=\{f+\bar{g}: f, g \in M\}
$$

is a self-conjugate subspace of $C(X)$. According to [7, Lemma 2.5] (or [3, Corollary 3.2]), there is a continuous surjection $\varphi: C h\left(T_{\infty}(M)\right) \longrightarrow C h(M)$ such that

$$
T_{\infty} f(y)=f(\varphi(y))\left(f \in M, y \in C h\left(T_{\infty}(M)\right)\right) .
$$

Since $C h\left(T_{\infty}(M)+\overline{T_{\infty}(M)}\right)=C h\left(T_{\infty}(M)\right)$ and $C h(M+\bar{M})=C h(M)$ (17, Lemma 2.3]) are boundaries, $T_{\infty}$ can be extended to a linear isometry $\widetilde{T}_{\infty}: M+\bar{M} \longrightarrow C(Y)$ such that

$$
\widetilde{T}_{\infty}(f+\bar{g})(y)=f(\varphi(y))+\overline{g(\varphi(y))}\left(f, g \in M, y \in C h\left(T_{\infty}(M)\right)\right) .
$$

Moreover, by [7, Lemma 3.2], each $T_{n}$ can be extended to a positive linear map $\widetilde{T}_{n}$ from $\bar{M}+M$ into $C(Y)$. Now, we get the result from Theorems 3.1 and 3.2 .

\section{EXAMPLES}

In this section we provide several examples which show how our results can be applied.

Example 4.1. Let $k \in \mathbb{N} \cup\{0, \infty\}$ and $C^{(k)}(I)$ denote the space of $k$-times continuously differentiable functions on the interval $I=[0,1]$ which is a self-conjugate space. Suppose that $\left\{T_{n}\right\}$ is a sequence of positive linear maps from $C^{(k)}(I)$ into $C(I)$ satisfying

$$
T_{n} 1 \longrightarrow 1, T_{n} x \longrightarrow x, T_{n} x^{2} \longrightarrow x^{2}
$$

For each $a \in I$, the function $h(x)=1-(x-a)^{2}$ belongs to the function space $S=\operatorname{Span}\left\{1, x, x^{2}\right\}$. Since $h(a)=1$ and $|h(y)|<1$ for any $y \neq a$, we infer $C h(S)=I$, by Remark 2.1. Now from Theorem 3.1 we conclude that $T_{n} f \longrightarrow f$ for all $f \in C^{(k)}(I)$. Meantime, by Theorem 3.2 the same result holds true for "uniformly convergence" instead of "pointwise convergence", which can be also obtained from Korovkin's first theorem. 
Example 4.2. Let $\Omega$ be a non-empty open subset of $\mathbb{R}^{p}$ and $K$ be a compact subset of $\Omega$. The term multi-index denotes an ordered $p$-tuple $\alpha=\left(\alpha_{1}, \ldots, \alpha_{p}\right)$ of nonnegative integers $\alpha_{i}$. For each multi-index $\alpha$, consider the differential operator

$$
D^{\alpha}=\left(\frac{\partial}{\partial x_{1}}\right)^{\alpha_{1}} \ldots\left(\frac{\partial}{\partial x_{p}}\right)^{\alpha_{p}}
$$

if $\alpha \neq 0$, and $D^{\alpha} f=f$ if $\alpha=0$. A function $f$ on $\Omega$ is said to belong to $C^{\infty}(\Omega)$ if $D^{\alpha} f \in C(\Omega)$ for all multi-index $\alpha$. By $\mathcal{D}_{K}$ we denote the space $\left\{\left.f\right|_{K}: f \in C^{\infty}(\Omega)\right\}$. Since $\mathcal{D}_{K}$ may be considered as a function space on $K$, from our results we deduce the following.

If $\left\{T_{n}: \mathcal{D}_{K} \longrightarrow C(K): n \in \mathbb{N}\right\}$ is a sequence of positive linear maps such that $T_{n} 1 \longrightarrow 1$, $T_{n}\left(P_{k}\right) \longrightarrow P_{k}, T_{n}\left(\sum_{k=1}^{p} P_{k}^{2}\right) \longrightarrow \sum_{k=1}^{p} P_{k}^{2}$, where $P_{k}$ is the projection

$$
P_{k}(x)=x_{k} \text { for } x=\left(x_{1}, \ldots, x_{p}\right),
$$

then $T_{n} f \longrightarrow f$ for all $f \in \mathcal{D}_{K}$. A similar result holds true for "uniformly convergence" instead of "pointwise convergence".

Let us remark that for any $a=\left(a_{1}, \ldots, a_{p}\right) \in K$, the function

$$
h(x)=b_{1}-\left(P_{1}(x)-a_{1}\right)^{2}+\ldots+b_{k}-\left(P_{p}(x)-a_{p}\right)^{2} \quad\left(x=\left(x_{1}, \ldots, x_{p}\right) \in \Omega\right),
$$

where $b_{i}>\max \left\{\left|P_{i}(x)-a_{i}\right|: x \in K\right\}, i=1, \ldots, p$, implies that $a$ belongs to the Choquet boundary of $S=\operatorname{Span}\left\{1, P_{1}, \ldots, P_{p}, P_{1}^{2}, \ldots, P_{p}^{2}\right\}$ by Remark 2.1 .

The following example includes the complex Korovkin theorem.

Example 4.3. If $\left\{T_{n}: C(\mathbb{T}) \longrightarrow C(\mathbb{T}): n \in \mathbb{N}\right\}$ is a sequence of positive linear maps such that $T_{n} 1 \longrightarrow 1$ and $T_{n} z \longrightarrow z$, then $T_{n} f \longrightarrow f$ for all $f \in C(\mathbb{T})$. Notice that here if $z_{0} \in \mathbb{T}$, then the function $h(z)=\frac{z+z_{0}}{2}$ works for Remark $2.1(S=\operatorname{Span}\{1, z\})$.

Let $D$ be the closed unit disc $\{z \in \mathbb{C}:|z| \leq 1\}$ and $\left\{T_{n}: C(D) \longrightarrow C(D): n \in \mathbb{N}\right\}$ be a sequence of positive linear maps such that $T_{n} 1 \longrightarrow 1, T_{n} z \longrightarrow z, T_{n}|z|^{2} \longrightarrow|z|^{2}$, then $T_{n} f \longrightarrow f$ for all $f \in C(D)$.

It should be noted that since $T_{n}$ is positive, it is easily seen that $T_{n} \bar{z}=\overline{T_{n} z}$, which yields $T_{n} \bar{z} \longrightarrow \bar{z}$. Hence for each $z_{0} \in D$, the function $h(z)=1-\frac{\left|z-z_{0}\right|^{2}}{4}=1-\frac{|z|^{2}-\bar{z} z_{0}-\overline{z_{0}} z+\left|z_{0}\right|^{2}}{4}$, which belongs to $S=\operatorname{Span}\left\{1, z, \bar{z},|z|^{2}\right\}$, is the appropriate function for Remark 2.1.

The two above results holds true for "uniformly convergence" instead of "pointwise convergence".

Remark 4.4. From our theorems, one can obtain the Korovkin-type results of [11] and [15] (with respect to both "uniformly convergence" and "pointwise convergence"), which are generalizations of Korovkin's second theorem on convergence of a sequence of positive linear maps for the space of real-valued continuous $2 \pi$-periodic functions on $\mathbb{R}$. 


\section{REFERENCES}

[1] F. Altomare, Korovkin-type theorems and approximation by positive linear operators, Surv. Approx. Theory. 5 (2010), 92-164.

[2] F. Altomare and M. Campiti, Korovkin-Type Approximation Theory and its Applications, de Gruyter Studies in Mathematics, 17, Walter de Gruyter Co., Berlin, 1994.

[3] J. Araujo and J. J. Font, Linear isometries between subspaces of continuous functions, Trans. Amer. Math. Soc. 349 (1997), 413-428.

[4] E. Bishop and K. de Leeuw, The representation of linear functionals by measures on sets of extreme points, Ann. Inst. Fourier (Grenoble) 9 (1959), 305-331.

[5] A. Browder, Introduction to Function Algebras, W. A. Benjamin, New York-Amsterdam, 1969.

[6] K. Donner, Extension of Positive Operators and Korovkin Theorems, Lecture Notes in Math. 904, SpringerVerlag, Berlin, 1982.

[7] T. Hachiro and T. Okayasu, Some theorems of Korovkin type, Studia Math. 155 (2003), no. 2, 131-143.

[8] P. P. Korovkin, On convergence of linear positive operators in the space of continuous functions, Doklady Akad. Nauk SSSR (N.S.) 90 (1953), 961-964 (in Russian).

[9] H. Koshimizu, T. Miura, H. Takagi and S. E. Takahasi, Real-linear isometries between subspaces of continuous functions, J. Math. Anal. Appl. 413 (2014), 229-241.

[10] S. Mazur and S. Ulam, Sur les transformations isométriques d'espaces vectoriels normés, C. R. Math. Acad. Sci. Paris 194 (1932), 946-948.

[11] E. N. Morozov, Convergence of a sequence of positive linear operators in the space of continuous 2 $\pi$-periodic functions of two variables, Kalinin. Gos. Ped. Inst. Uchen. Zap. 26 (1958), 129-142 (in Russian).

[12] R. R. Phelps, Lectures on Choquet's Theorem, 2nd ed., Lecture Notes in Math. 1757, Springer, Berlin, 2001.

[13] R. R. Phelps, The range of Tf for certain linear operators T, Proc. Amer. Math. Soc. 16 (1965), 381-382.

[14] E. Scheffold, Uber die punktweise konvergenz von operatoren in $C(X)$, Rev. Acad. Ci. Zaragoza 28 (1973), $5-12$.

[15] V. I. Volkov, Conditions for convergence of a sequence of positive linear operators in the space of continuous functions of two variables, Kalinin. Gos. Ped. Inst. Uchen. Zap. 26 (1958), 27-40 (in Russian).

Department of Mathematics, K. N. Toosi University of Technology, Tehran, 16315-1618, Iran

E-mail address: m.hosseini@kntu.ac.ir

Departamento de Matemáticas, Universitat Jaume i, Campus Riu Sec, 8029 AP, Castellón, Spain

E-mail address: font@mat.uji.es 